\title{
CLASH-VLT: CONSTRAINTS ON THE DARK MATTER EQUATION OF STATE FROM ACCURATE MEASUREMENTS OF GALAXY CLUSTER MASS PROFILES
}

\author{
Barbara Sartoris ${ }^{1,2,3}$, Andrea Biviano ${ }^{2}$, Piero Rosati ${ }^{4}$, Stefano Borgani $^{1,2,3}$, Keilchi Umetsu ${ }^{5}$, \\ Matthias BartelmanN ${ }^{6}$, Marisa Girardi ${ }^{1,2}$, Claudio Grillo ${ }^{7}$, Doron Lemze $^{8}$, Adi Zitrin ${ }^{9,26}$, Italo Balestra ${ }^{2,10}$, \\ amata Mercurio ${ }^{10}$, Mario Nonino ${ }^{2}$, Marc Postman ${ }^{11}$, Nicole CZakon $^{5}$, Larry Bradley ${ }^{11}$, Tom Broadhurst ${ }^{12}$, \\ Dan Coe ${ }^{11}$, Elinor Medezinski ${ }^{8}$, Peter Melchior ${ }^{13}$, Massimo Meneghetti ${ }^{14,15}{ }^{15}$ Julian Merten $^{16}$, \\ Marianna Annunziatella ${ }^{1,2}$, Narciso Benitez ${ }^{17}$, Oliver Czoske $^{18}$, Megan Donahue ${ }^{19}$, STtefano Ettori $^{14,15}$, \\ Holland Ford ${ }^{8}$, Alexander Fritz ${ }^{20}$, Dan Kelson ${ }^{21}$, Anton Koekemoer ${ }^{11}$, Ulrike Kuchner ${ }^{18}$, Marco Lombardi ${ }^{22}$, \\ Christian Maier $^{18}$, Leonidas A. Moustakas $^{16}$, Emiliano Munari ${ }^{1,2}$, Valentina Presotto $^{1,2}$, Marco ScodegGio $^{20}$, \\ Stella Seitz ${ }^{23,24}$, PaOlo Tozzi ${ }^{25}$, Wei Zheng ${ }^{8}$, and Bodo Ziegler ${ }^{18}$ \\ ${ }^{1}$ Dipartimento di Fisica, Sezione di Astronomia, Università di Trieste, Via Tiepolo 11, I-34143 Trieste, Italy; sartoris@oats.inaf.it \\ ${ }^{2}$ INAF/Osservatorio Astronomico di Trieste, Via Tiepolo 11, I-34143 Trieste, Italy \\ ${ }^{3}$ INFN, Sezione di Trieste, Via Valerio 2, I-34127 Trieste, Italy \\ ${ }^{4}$ Dipartimento di Fisica e Scienze della Terra, Universita' di Ferrara, Via Saragat 1, I-44122 Ferrara, Italy \\ 5 Institute of Astronomy and Astrophysics, Academia Sinica, P.O. Box 23-141, Taipei 10617, Taiwan \\ ${ }^{6}$ Zentrum für Astronomie der Universität Heidelberg, ITA, Albert-Ueberle-Str. 2, D-69120 Heidelberg, Germany \\ ${ }^{7}$ Dark Cosmology Centre, Niels Bohr Institute, University of Copenhagen, Juliane Maries Vej 30, DK-2100 Copenhagen, Denmark \\ ${ }^{8}$ Department of Physics and Astronomy, The Johns Hopkins University, 3400 North Charles Street, Baltimore, MD 21218, USA \\ ${ }^{9}$ Cahill Center for Astronomy and Astrophysics, California Institute of Technology, MS 249-17, Pasadena, CA 91125, USA \\ ${ }^{10}$ INAF/Osservatorio Astronomico di Capodimonte, Via Moiariello 16, I-80131 Napoli, Italy \\ ${ }^{11}$ Space Telescope Science Institute, 3700 San Martin Drive, Baltimore, MD 21218, USA \\ ${ }^{12}$ Department of Theoretical Physics, University of the Basque Country, P.O. Box 644, E-48080 Bilbao, Spain \\ ${ }^{13}$ Department of Physics, The Ohio State University, Columbus, OH 43210, USA \\ ${ }^{14}$ INAF/Osservatorio Astronomico di Bologna, Via Ranzani 1, I-40127 Bologna, Italy \\ ${ }^{15}$ INFN, Sezione di Bologna, Via Ranzani 1, I-40127 Bologna, Italy \\ ${ }^{16}$ Jet Propulsion Laboratory, California Institute of Technology, 4800 Oak Grove Drive, Pasadena, CA 91109, USA \\ ${ }^{17}$ Instituto de Astrofísica de Andalucía (CSIC), C/Camino Bajo de Huétor 24, E-18008 Granada, Spain \\ ${ }^{18}$ Department of Astrophysics, University of Vienna, Türkenschanzstr. 17, A-1180 Wien, Austria \\ ${ }^{19}$ Department of Physics and Astronomy, Michigan State University, East Lansing, MI 48824, USA \\ ${ }^{20}$ INAF/IASF-Milano, Via Bassini 15, I-20133 Milano, Italy \\ ${ }^{21}$ Observatories of the Carnegie Institution of Washington, Pasadena, CA 91101, USA \\ ${ }^{22}$ Dipartimento di Fisica, Universitá degli Studi di Milano, Via Celoria 16, I-20133 Milan, Italy \\ ${ }^{23}$ University Observatory Munich, Scheinerstrasse 1, D-81679 München, Germany \\ ${ }^{24}$ Max-Planck-Institut für Extraterrestrische Physik, Postfach 1312, Giessenbachstrasse, D-85741 Garching, Germany \\ ${ }^{25}$ INAF/Osservatorio Astrofisico di Arcetri, Largo E. Fermi 5, I-50125 Firenze, Italy \\ Received 2013 November 18; accepted 2014 January 21; published 2014 February 14
}

\begin{abstract}
A pressureless scenario for the dark matter (DM) fluid is a widely adopted hypothesis, despite the absence of direct observational evidence. According to general relativity, the total mass-energy content of a system shapes the gravitational potential well, but different test particles perceive this potential in different ways depending on their properties. Cluster galaxy velocities, being $\ll c$, depend solely on the gravitational potential, whereas photon trajectories reflect the contributions from the gravitational potential plus a relativistic-pressure term that depends on the cluster mass. We exploit this phenomenon to constrain the equation of state (EoS) parameter of the fluid, primarily DM, contained in galaxy clusters. We use complementary information provided by the kinematic and lensing mass profiles of the galaxy cluster MACS $1206.2-0847$ at $z=0.44$, as obtained in an extensive imaging and spectroscopic campaign within the Cluster Lensing And Supernova survey with Hubble. The unprecedented high quality of our data set and the properties of this cluster are well suited to determine the EoS parameter of the cluster fluid. Since baryons contribute at most $15 \%$ to the total mass in clusters and their pressure is negligible, the EoS parameter we derive describes the behavior of the DM fluid. We obtain the most stringent constraint on the DM EoS parameter to date, $w=\left(p_{r}+2 p_{t}\right) /\left(3 c^{2} \rho\right)=0.00 \pm 0.15$ (stat) \pm 0.08 (syst), averaged over the radial range $0.5 \mathrm{Mpc} \leqslant r \leqslant r_{200}$, where $p_{r}$ and $p_{t}$ are the radial and tangential pressure, and $\rho$ is the density. We plan to further improve our constraint by applying the same procedure to all clusters from the ongoing Cluster Lensing And Supernova Survey with Hubble-Very Large Telescope program.
\end{abstract}

Key words: cosmology: theory - dark matter - equation of state - galaxies: clusters: general galaxies: clusters: individual (1206.2-0847)

\section{INTRODUCTION}

In the standard cosmological scenario, non-baryonic dark matter (DM) plays a decisive role, representing approximately

\footnotetext{
${ }^{26}$ Hubble Fellow.
}

$27 \%$ of the mass-energy content of the universe (Planck Collaboration et al. 2013). The existence and properties of weakly interacting massive DM particles are inferred from mass measurements of galaxies and galaxy clusters derived from kinematics, gravitational lensing, or other probes. Additional indirect evidences come from the influence DM has on the 
formation and evolution of the large-scale structure of the universe, and from its effects on the temperature anisotropies observed in the cosmic microwave background (e.g., Peebles 1980).

A widely adopted assumption in cosmology is that DM is a pressureless fluid. Although such hypothesis has not been directly proven yet, in a universe where the DM fluid has a large pressure, there would not be enough time for cosmological structures to grow between the recombination epoch and today (e.g., Coles \& Lucchin 2002). Moreover, the observed properties of the large-scale structure are consistent with the current pressureless DM scenario (e.g., Sánchez et al. 2012, 2013; Samushia et al. 2013).

Despite considerable efforts for a direct detection of DM with underground experiments (e.g., DAMA, ${ }^{27} \mathrm{XENON},{ }^{28}$ PICASSO, ${ }^{29} \mathrm{XMASS}^{30} \mathrm{CDMS}^{31}$ ) no significant signal has been found to date and the nature of DM remains unknown.

The current pressureless scenario sets the DM equation of state $(\mathrm{EoS})$ parameter to be zero, by definition. The aim of this Letter is to directly test this assumption by constraining the EoS parameter of the galaxy cluster fluid, using new accurate measurements of a cluster mass profile from lensing and kinematics analyses.

Bharadwaj \& Kar (2003) first proposed combining the analyses of the rotational curves and of the lensing signal in spiral galaxies to constrain the amount of DM pressure. Faber \& Visser (2006) generalized the Bharadwaj \& Kar (2003) approach without assuming any model for both the DM EoS and the rotational curves. Finally, Serra \& Domínguez Romero (2011) extended the method to the case of galaxy clusters.

In this Letter, we apply this method to the $z=0.44$ cluster MACS 1206.2-0847 (hereafter MACS 1206), which has been studied in detail as part of the Cluster Lensing And Supernova survey with Hubble (CLASH; Postman et al. 2012). Umetsu et al. (2012) used new high quality imaging of this cluster obtained with Subaru and the Hubble Space Telescope (HST), to derive its mass density profile from weak-lensing distortion, magnification, and strong-lensing analyses. Biviano et al. (2013) (hereafter B13) used an unprecedented data set of about 600 measured redshifts of cluster members, obtained as part of a VLT/VIMOS Large Programme (ID 186.A-0798) to determine the cluster mass profile over the radial range of $0.05-2.5$ virial radii by applying the Jeans equation (Mamon et al. 2013) and the caustic technique of Diaferio \& Geller (1997).

By using complementary information provided by the kinematics and lensing mass profile determinations, in this Letter we constrain the DM EoS parameter which encapsulates information on the amount of fluid pressure.

\section{THEORETICAL FRAMEWORK}

A static, spherically symmetric spacetime is described by the metric (e.g., Misner et al. 1973):

$$
d s^{2}=-e^{2 \Phi(r) / c^{2}} d t^{2}+e^{2 \lambda(r)} d r^{2}+r^{2} d \theta^{2}+r^{2} \sin ^{2} \theta d \varphi^{2},
$$

where $\Phi(r)$ and $\lambda(r)$ are two arbitrary generic functions of the metric. One can apply to such a metric the Einstein

\footnotetext{
27 http://people.roma2.infn.it/ dama/web/home.html

$28 \mathrm{http}: / /$ xenon.astro.columbia.edu/

29 http://www.picassoexperiment.ca/

30 http://www-sk.icrr.u-tokyo.ac.jp/xmass/index-e.html

31 http://cdms.berkeley.edu/
}

field equations, $G_{\alpha \beta}=\left(8 \pi G / c^{4}\right) T_{\alpha \beta}$, by using the stressenergy tensor of an ideal gas with radial, $T_{r r}$, and tangential, $T_{\theta \theta}$, pressure components: $T_{\alpha \beta}=\left(\rho+p / c^{2}\right) u_{\alpha} u_{\beta}+p g_{\alpha \beta}$. In these equations, $G_{\alpha \beta}$ is the Einstein tensor, $G$ is Newton's gravitational constant, $c$ is the speed of light in vacuum, $u_{\alpha}$ is the fluid's four velocity, and $g_{\alpha \beta}$ is the metric tensor.

It is then possible to obtain the density profile and the pressure profiles in the radial and transverse directions (e.g., Schutz 2009):

$$
\begin{aligned}
\rho(r)= & \frac{1}{4 \pi} \frac{m^{\prime}(r)}{r^{2}}, \\
p_{r}(r)= & -\frac{c^{4}}{8 \pi G} \frac{2}{r^{2}}\left[\frac{G m(r)}{c^{2} r}-r \frac{\Phi^{\prime}(r)}{c^{2}}\left(1-\frac{2 G m(r)}{c^{2} r}\right)\right], \\
p_{t}(r)= & \frac{c^{4}}{8 \pi G}\left\{\left(1-\frac{2 G m(r)}{c^{2} r}\right) \frac{1}{c^{2}}\left[\frac{\Phi^{\prime}(r)}{r}+\frac{\Phi^{\prime}(r)^{2}}{c^{2}}+\Phi^{\prime \prime}(r)\right]\right. \\
& \left.-\frac{G}{c^{2}}\left(\frac{m(r)}{r}\right)^{\prime}\left(\frac{1}{r}+\frac{\Phi^{\prime}(r)}{c^{2}}\right)\right\},
\end{aligned}
$$

where the prime denotes the partial derivative with respect to the $r$-coordinate, $m(r)$ is the mass within a sphere of radius $r$, and the metric function $e^{2 \lambda(r)}$ is defined as

$$
e^{-2 \lambda(r)} \triangleq 1-\frac{2 G m(r)}{c^{2} r} .
$$

For a medium with isotropic pressure, the metric Equation (1) (describing the geometry of the spacetime inside the cluster) matches the Schwarzschild metric (describing the geometry of the spacetime outside the cluster) at $r=\tilde{r}$, where $\tilde{r}$ is the size of the cluster, only if $p_{r}=p_{t}=0$ (Bharadwaj \& Kar 2003).

Thus, to check the validity of the pressureless assumption, throughout this Letter, we consider a fluid with anisotropic pressure and the most general definition of EoS:

$$
w(r)=\frac{p_{r}(r)+2 p_{t}(r)}{3 c^{2} \rho(r)} .
$$

The functions $\Phi(r)$ and $m(r)$ fully characterize the metric and the density and pressure profiles, and, consequently, the EoS. We calculate the two metric functions by using the mass profiles of the cluster derived from the velocity distribution of cluster galaxies and from gravitational lensing measurements.

In general relativity, the mass-energy content of the cluster shapes the gravitational potential well. However, the two probe particles used in the aforementioned analyses, the galaxies and the photons, perceive the gravitational potential in different ways, due to their distinct properties.

To calculate the trajectory of the galaxy test particles, with velocity $v \ll c$ (B13 measured the velocity dispersion of the MACS 1206 cluster $\sigma_{\mathrm{los}}=1087_{-55}^{+53} \mathrm{~km} \mathrm{~s}^{-1}$ ), we relate Einstein's field equations to the Poisson equation in the weak field approximation $\left(2 \Phi \ll c^{2}\right.$ and $\left.2 m G / r \ll c^{2}\right)$. Thus, the $(0,0)$ component of Einstein's field equations reads

$$
R_{00} \approx \nabla^{2} \Phi=\frac{4 \pi G}{c^{2}}\left(c^{2} \rho+p_{r}+2 p_{t}\right)
$$

for the cluster fluid (e.g., Schutz 2009), where the metric potential $\Phi$ is different from $\Phi_{N}$, the Newtonian potential. In the Newtonian limit, $\rho \gg p / c^{2}$, and we recover the usual Poisson equation $\nabla^{2} \Phi_{N}=4 \pi G \rho$. 
From Equation (7), we can see that the mass profile derived from the kinematics analysis, $m_{k}(r)$, depends only on the $(0,0)$ part of the metric. Therefore the relation between the Poisson equation (Equation (7)) and this mass profile is

$$
m_{k}(r)=\frac{r^{2}}{G} \nabla \Phi(r) .
$$

Since, in the lensing analysis, the probe particles travel at the speed of light, full treatment of the geodesics is needed, even in the case of weak field approximation. Misner et al. (1973; see also Faber \& Visser 2006) derived the lensing potential by applying Fermat's principle to the geodesics of the photons moving through the cluster potential described by the metric Equation (1). In this context, the light-ray trajectory is fully described by the relativistic analog of the refractive index. For the metric Equation (1), the effective refractive index in the weak field approximation is

$$
\begin{aligned}
n(r)=1 & -\frac{\Phi(r)}{c^{2}}-\frac{G}{c^{2}} \int \frac{m(r)}{r^{2}} d r \\
& +O\left[\left(\frac{2 G m}{c^{2} r}\right)^{2}, \frac{2 G m}{r} \frac{\Phi}{c^{2}}, \frac{\Phi^{2}}{c^{4}}\right]
\end{aligned}
$$

and it is possible to define the lensing potential as

$$
2 \Phi_{l}(r)=\Phi(r)+G \int \frac{m(r)}{r^{2}} d r .
$$

The trajectory of a light particle is determined entirely by the effective refractive index $n(r)$, thus the bending of light is not only due to $\Phi(r)$ (see Equation (7)), but also to an extra term due to the relativistic pressure of the fluid.

The mass profile derived from the lensing analysis can be related to the lensing potential through the Poisson equation:

$$
\begin{gathered}
\rho_{l}(r)=\frac{1}{4 \pi G} \nabla^{2} \Phi_{l}(r), \\
m_{l}(r)=\frac{r^{2}}{G} \Phi_{l}^{\prime}(r)=\frac{r^{2}}{2 G} \Phi^{\prime}(r)+\frac{m(r)}{2}=\frac{m_{k}(r)}{2}+\frac{m(r)}{2} .
\end{gathered}
$$

Note that, while according to Equation (7) the observed galaxy kinematics depends on the metric component $g_{00}$ alone, the observed gravitational lensing potential reflects contributions from both $g_{00}$ and $g_{r r}$. Using the kinematic and lensing mass profiles, we can finally determine the two metric functions $\Phi(r)$ (from Equation (8)) and $m(r)$ (from Equation (12)) and calculate the EoS of the cluster fluid (Equation (6)).

\section{THE MACS 1206 CLUSTER MASS PROFILES}

The X-ray selected MACS 1206 cluster, at redshift 0.44, has been observed in the course of the CLASH survey. HST observations were completed in 2011. A detailed strong-lensing model, based on the identification of 50 multiple lensed images of 13 background galaxies, was presented by Zitrin et al. (2012). The combination of the inner mass density profile from this model with weak-lensing shear and magnification measurements from Subaru multi-band images led to a reliable determination of the mass density profile of MACS 1206 out to $\sim 2 \mathrm{Mpc}^{32}$ (Umetsu et al. 2012).

\footnotetext{
$\overline{32 \text { Umetsu et al. (2012) and B13 adopted } \Omega_{m}}=0.3, \Omega_{\Lambda}=0.7, h=0.7$.
}

The spectroscopic observations with the Very Large Telescope (VLT), which led to a total of 2749 objects with reliable redshift measurements in the cluster field, and the kinematic analysis, are described in B13. Using the projected phase-space distribution of these objects and several techniques for the rejection of interlopers, 592 cluster members were identified. This large spectroscopic sample was used to determine the kinematic mass profile out to the virial radius $(\sim 2 \mathrm{Mpc})$ by solving the Jeans equation with the MAMPOSSt (Mamon et al. 2013) technique, and further extended to $5 \mathrm{Mpc}$ with the caustic method of Diaferio \& Geller (1997).

The kinematic determination of the cluster mass profile is in very good agreement both with the lensing determination and with that based on X-ray Chandra observations, under the assumption of hydrostatic equilibrium (see Umetsu et al. 2012, B13).

The fact that different probes of the cluster mass profile converge to similar results ${ }^{33}$ suggests that systematic effects in the mass determination are negligible and that the cluster is dynamically relaxed. Moreover, the analysis of Lemze et al. (2013) did not find a significant level of substructure within this cluster when using the most conservative membership selection. The concentric distribution of different mass components (DM, stellar light, and gas) also underscores an equilibrium configuration. All these properties make MACS 1206 an ideal candidate for testing the EoS of the cluster fluid.

Umetsu et al. (2012) parameterized the lensing mass profile of MACS 1206 with the Navarro-Frenk-White (NFW) model (Navarro et al. 1997). The same model was found by B13 to provide the highest likelihood fit to the kinematic data. We therefore use the NFW model parameterization of the kinematic and lensing mass profiles in our analyses. To check the sensitivity of our results on the kinematic mass profile used, we also consider the Hernquist (1990) and Burkert (1995) profiles. These models are quite different from NFW and yet were found to provide acceptable fits to the kinematic data (B13).

Unlike the lensing determination of the cluster mass profile, the kinematic determination also requires modeling the velocity anisotropy profile of the tracers of the gravitational potential, $\beta(r)$. B13 considered three possible ansatz models, named " $\mathrm{O}$,"

$$
\beta_{\mathrm{O}}(r)=\beta_{\infty} \frac{r-r_{-2}}{r+r_{-2}},
$$

“T” (from Tiret et al. 2007),

$$
\beta_{\mathrm{T}}(r)=\beta_{\infty} \frac{r}{r+r_{-2}},
$$

and "C" with a constant anisotropy with radius. In Equations (14) and (15), $r_{-2}$ is the scale radius at which the logarithmic derivative of the mass density profile equals -2 , $d \ln \rho / d \ln r=-2$ and $\beta_{\infty}$ is the anisotropy value at large radii. The $\mathrm{O}$ model gives the smallest product of relative errors in the two free parameters of the mass profile $\left(r_{200}{ }^{34}\right.$ and $\left.r_{-2}\right)$, namely,

\footnotetext{
33 Note that the similarity of the kinematic and lensing mass profiles does not imply a pressureless fluid $(w(r)=0)$, since, on first approximation, $w(r)$ does not depend on the profiles, but on their derivatives

$$
w(r) \approx \frac{2}{3} \frac{m_{k}^{\prime}(r)-m_{l}^{\prime}(r)}{2 m_{l}^{\prime}(r)-m_{k}^{\prime}(r)}
$$

(Faber \& Visser 2006).

34 The radius $r_{200}$ is the radius of a sphere with mass overdensity $\Delta=$ 200 times the critical density of the universe at the cluster redshift. We use $r_{200} \simeq 2 \mathrm{Mpc}$ from Umetsu et al. (2012) and B13.
} 


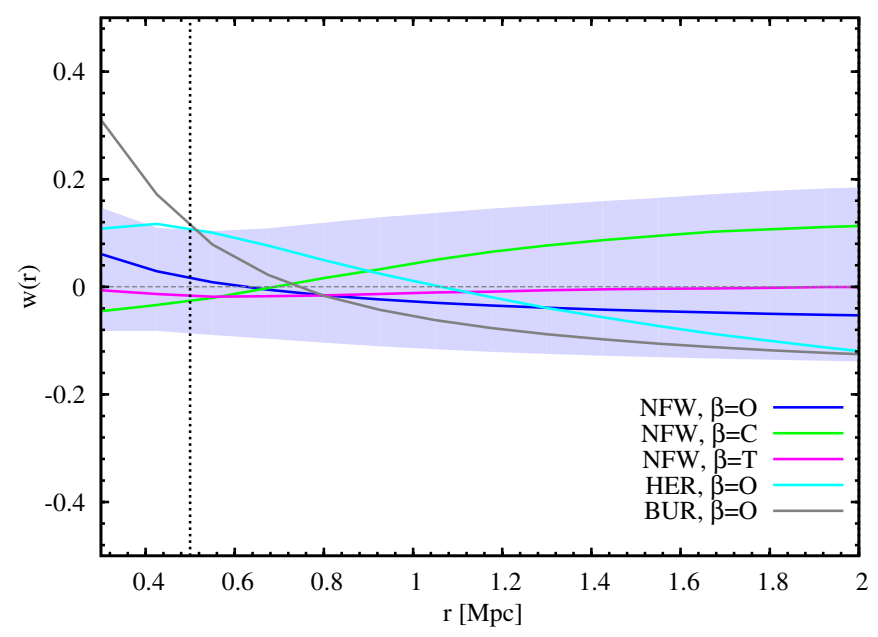

Figure 1. EoS parameter of the cluster fluid as a function of clustercentric radius obtained by using different prescriptions for the mass and the anisotropy profiles $(\beta)$ in the kinematic analysis: NFW $\beta=\mathrm{O}$ (solid blue curve), NFW $\beta=\mathrm{C}$ (solid green curve), NFW $\beta=\mathrm{T}$ (solid magenta curve), Burkert $\beta=$ $\mathrm{O}$ (solid gray curve), Hernquist $\beta=\mathrm{O}$ (solid cyan curve). The shaded area represents the errors for our reference model $(\mathrm{NFW}+\mathrm{O})$, calculated through a Monte Carlo sampling method. Errors on the other models are not shown for the sake of clarity. The vertical dotted line indicates the lower radial limit above which mean $w$ values in Table 1 are computed.

it maximizes the ratio $\left(r_{200} r_{-2}\right) /\left(\delta r_{200} \delta r_{-2}\right)$, where $\delta r_{200}$ and $\delta r_{-2}$ are the (symmetrized) errors on $r_{200}$ and $r_{-2}$, respectively. Therefore, the NFW+O model is adopted as our reference model in the following analysis.

\section{RESULTS}

In this section, we present and discuss our results on the EoS of the DM fluid as obtained from the analysis of the lensing and kinematic mass profiles of MACS 1206 described above. Derivatives of the potential and the mass profiles in Equations (2)-(4) are computed directly from the models of Umetsu et al. (2012) and B13. For details see Sections 2 and 3.

In Figure 1, we show the resulting EoS parameter $w(r)$ (Equation (6)) as a function of the clustercentric radius. The statistical errors have been calculated via $10^{4}$ Monte Carlo resamplings by propagating the uncertainties on the parameters of the lensing and kinematic mass profile models, $r_{200}$ and $r_{-2}$. As for the parameters derived from kinematics analysis, B13 shows that $r_{200}$ and $r_{-2}$ have uncorrelated errors, thus we use the probability distributions shown in Figure 9 of B13 to explore the parameter space in the Monte Carlo sampling. Umetsu et al. (2012) shows that the joint weak- and strong-lensing contours of the mass profile model are elliptical, so we can assume that they are Gaussian distributed but with covariance between the model parameters. We perform a Monte Carlo Markov Chain fitting to the weak- and strong-lensing radial profiles to generate posterior probability distribution functions in $r_{200}$ and $r_{-2}$ to consider the covariance between these two parameters in the calculation of the errors on the EoS parameter profile.

In Figure 1, the shaded area indicates the error computed from the 16th and 84th percentiles of the probability distribution at varying radii, for the reference $\mathrm{NFW}+\mathrm{O}$ model. In Table 1, we list the mean values of $w$, calculated over the radial range $0.5 \mathrm{Mpc} \leqslant r \leqslant r_{200}$, where $w(r)$ is approximately constant. Since the errors on the EoS parameter at different radii, $w(r)$, are highly correlated, we list $w$ errors in Table 1 obtained by computing the average of the $w$ uncertainties over the
Table 1

Mean Values and Errors for the EoS Parameter of the Cluster Fluid

\begin{tabular}{lccc}
\hline \hline Profiles & $\beta$ & $w$ & $\Delta w$ \\
\hline NFW & O & $\mathbf{0 . 0 0}$ & $\mathbf{0 . 1 5}$ \\
NFW & $\mathrm{C}$ & 0.15 & 0.19 \\
NFW & T & 0.08 & 0.18 \\
BUR & O & -0.01 & 0.15 \\
HER & O & 0.06 & 0.19 \\
\hline
\end{tabular}

Notes. The mean values are obtained within the radial range $0.5 \mathrm{Mpc} \leqslant r \leqslant r_{200}$, using different prescriptions of the mass profile and anisotropy parameter in the kinematic analysis. Column 1: mass density profiles. Column 2: anisotropy parameter. Column 3: mean values for the EoS parameter. Column 4: average of the errors on $w(r)$ over the considered radial range. The reference model is indicated in bold.

considered radial range. We limit the radial range to radii $\leqslant r_{200}$ because at larger radii both the kinematics and the lensing determinations of the cluster mass profile might be affected by systematics (deviation from dynamical relaxation for the kinematics and contamination by a large-scale structure filament for the lensing). Moreover, the DM, whose EoS we want to constrain, dominates the mass budget in this radial range.

Both in the figure and in the table, we show how our results are modified when we use different models for the anisotropy and kinematic mass profiles. The results on $w(r)$ are sensitive to the adopted models, but still within the statistical uncertainties of the reference models.

In Table 1, all the mean values are consistent with zero, and thus with the usually adopted pressureless assumption. Note that the mean values cannot be directly inferred from the curves of Figure 1 because the errors are asymmetric. In particular, for the reference $\mathrm{NFW}+\mathrm{O}$ model, we find a radially averaged value

$$
w=0.00 \pm 0.15 \text { (stat) } \pm 0.08 \text { (syst) }
$$

where the statistical error is listed in Table 1 and the systematic error reflects the peak to peak variation in the mean values of $w$ obtained using different mass and anisotropy models.

In our estimate, we cannot disentangle the mass profile of the baryons from that of the DM; however, baryons contribute at most $15 \%$ of the total cluster mass at all radii (Biviano \& Salucci 2006). Moreover, we estimate that the baryon contribution to $w$ is $\sim 10^{-5}$. This is found by using the EoS of an ideal gas for the hot intracluster medium. We can therefore assume that the EoS parameter found in our study describes the behavior of the DM fluid in the cluster.

A previous analysis of the DM EoS (Serra \& Domínguez Romero 2011) found tentative evidence for a negative value of $w(r)$. This result was obtained by using the lensing and kinematic mass determinations of two clusters: Coma and CL0024+1654, which, however, are known to contain major substructures (Adami et al. 2005; Czoske et al. 2002), and their presence could affect the kinematic mass profile determinations. In particular, CL0024+1654 is possibly in a post-merger state after the core passage of two clusters occurring along the line of sight (Czoske et al. 2002; Umetsu et al. 2010). In any case, our constraints on $w$ are significantly more precise (by a factor of ¿3) than those obtained by Serra \& Domínguez Romero (2011) thanks to the significantly better quality of both our lensing and kinematic data. 


\section{SUMMARY AND CONCLUSIONS}

In this Letter, we discussed how the pressureless assumption for the DM fluid can be quantitatively verified and we obtained the most stringent constraint on the DM EoS available to date using high quality kinematic and lensing mass analyses of the relaxed CLASH cluster MACS 1206.

We confirmed the pressureless assumption, namely, $w=$ $0.00 \pm 0.15$ (stat) \pm 0.08 (syst). We find no radial dependence of $w$ outside the central $(0.5 \mathrm{Mpc})$ region.

The CLASH-VLT final sample will provide accurate mass profiles for 12 clusters, allowing us to place stronger constraints on the DM EoS parameter by stacking the information from all clusters. This will reduce our statistical errors, and, most importantly, possible systematic effects in the mass profile determinations such as departure from dynamical equilibrium and contamination by large-scale structure along the line of sight.

If departure from sphericity of the cluster potential well is detected, it is still possible, in principle, to apply the method used in this Letter to calculate the DM EoS parameter. Faber \& Visser (2006) showed how, given a mass distribution, it is always possible to recover the corresponding density and pressure distributions in absence of any particular potential symmetry and thus calculate the DM EoS parameter. From an observational point of view, cluster orientation and asphericity can systematically affect the mass profile determinations and consequently the EoS parameter. It is possible to reduce the impact of these errors by stacking results derived from a large sample of clusters.

We thank the referee, Michael Strauss, for his constructive and thoughtful comments. B.S. thanks Marino Mezzetti and Pierluigi Monaco for useful discussions. This work has been partially supported by the PRIN-MIUR09 "Tracing the Growth of Structures in the Universe," by the PD51 INFN grant and by the PRIN INAF 2010 "Architecture and Tomography of Galaxy Clusters." P.R. acknowledges partial support by the DFG Cluster of Excellence Origin and Structure of the Universe (http://www.universe-cluster.de). Support for A.Z. was provided by NASA through Hubble Fellowship grant HST-HF-51334.01-A awarded by STScI. A.F. acknowledges the support by INAF through PRIN 2008 (VIPERS) and PRIN 2010 (VIPERS) grants. This research was carried out in part at the Jet Propulsion Laboratory, California Institute of Technology, under a contract with NASA.

\section{REFERENCES}

Adami, C., Biviano, A., Durret, F., \& Mazure, A. 2005, A\&A, 443, 17 Bharadwaj, S., \& Kar, S. 2003, PhRvD, 68, 023516

Biviano, A., Rosati, P., Balestra, I., et al. 2013, A\&A, 558, A1

Biviano, A., \& Salucci, P. 2006, A\&A, 452, 75

Burkert, A. 1995, ApJL, 447, L25

Coles, P., \& Lucchin, F. 2002, Cosmology: The Origin and Evolution of Cosmic Structure (2nd ed.; New York: Wiley)

Czoske, O., Moore, B., Kneib, J.-P., \& Soucail, G. 2002, A\&A, 386, 31

Diaferio, A., \& Geller, M. J. 1997, ApJ, 481, 633

Faber, T., \& Visser, M. 2006, MNRAS, 372, 136

Hernquist, L. 1990, ApJ, 356, 359

Lemze, D., Postman, M., Genel, S., et al. 2013, ApJ, 776, 91

Mamon, G. A., Biviano, A., \& Boué, G. 2013, MNRAS, 429, 3079

Misner, C. W., Thorne, K. S., \& Wheeler, J. A. 1973, Gravitation (San Francisco, CA: Freeman)

Navarro, J. F., Frenk, C. S., \& White, S. D. M. 1997, ApJ, 490, 493

Peebles, P. J. E. 1980, The Large-Scale Structure of the Universe (Research Supported by the National Science Foundation; Princeton, NJ: Princeton Univ. Press), 435

Planck Collaboration, Ade, P. A. R., Aghanim, N., et al. 2013, arXiv:1303.5076

Postman, M., Coe, D., Benítez, N., et al. 2012, ApJS, 199, 25

Samushia, L., Reid, B. A., White, M., et al. 2013, MNRAS, 429, 1514

Sánchez, A. G., Kazin, E. A., Beutler, F., et al. 2013, MNRAS, 433, 1202

Sánchez, A. G., Scóccola, C. G., Ross, A. J., et al. 2012, MNRAS, 425, 415

Schutz, B. 2009, A First Course in General Relativity (Cambridge: Cambridge Univ. Press)

Serra, A. L., \& Domínguez Romero, M. J. L. 2011, MNRAS, 415, L74

Tiret, O., Combes, F., Angus, G. W., Famaey, B., \& Zhao, H. S. 2007, A\&A, 476, L1

Umetsu, K., Medezinski, E., Broadhurst, T., et al. 2010, ApJ, 714, 1470

Umetsu, K., Medezinski, E., Nonino, M., et al. 2012, ApJ, 755, 56

Zitrin, A., Rosati, P., Nonino, M., et al. 2012, ApJ, 749, 97 